\title{
Influence of Altitude on Caffeine, 5-Caffeoylquinic Acid, and Nicotinic Acid Contents of Arabica Coffee Varieties
}

\author{
Bealu Girma, ${ }^{1,2}$ Abera Gure $\mathbb{D}^{1}{ }^{1}$ and Feyisa Wedajo ${ }^{1}$ \\ ${ }^{1}$ Jimma University, College of Natural Science, Department of Chemistry, P.O. Box 378, Jimma, Ethiopia \\ ${ }^{2}$ Ethiopian Institute of Agricultural Research, Jimma Agricultural Research Center, P.O. Box 192, Jimma, Ethiopia \\ Correspondence should be addressed to Abera Gure; aberagure@gmail.com
}

Received 20 April 2020; Revised 29 June 2020; Accepted 13 July 2020; Published 12 August 2020

Academic Editor: Claudia Crestini

Copyright (c) 2020 Bealu Girma et al. This is an open access article distributed under the Creative Commons Attribution License, which permits unrestricted use, distribution, and reproduction in any medium, provided the original work is properly cited.

The influence of altitude on caffeine, 5-caffeoylquinic acid (5-CQA), and nicotinic acid contents of Arabica coffee varieties grown in Southwest Ethiopia was investigated. High-performance liquid chromatography with diode array detector (HPLC-DAD) was used for the determination of the target analytes. Coffee samples were collected from four coffee varieties, named as 74112, 7454, 7440, and 74110, which are cultivated in high, mid, and low altitudes in the study area. The findings of the study showed that the contents of caffeine and 5-CQA in both raw and roasted coffee beans decrease as the growing altitude increases and, thus, for all varieties, their highest concentrations were recorded in lowland coffee beans. Nevertheless, the contents of nicotinic acid increase as the altitude rises and, thus, the highest nicotinic acid content was recorded in highland coffee samples. Besides, after roasting, the contents of caffeine were increased, whereas the contents of 5-CQA were lowered, indicating the possibility of its degradation during the roasting process. Both green and roasted coffee beans also contained relatively higher concentrations of nicotinic acids. Other than the growing altitudes, the contents of caffeine, 5-CQA, and nicotinic acid in coffee beans also vary with coffee varieties. Therefore, coffee varieties that are cultivated at various altitudes may have different biochemical compositions such as caffeine, CGAs, and nicotinic acid that could greatly influence the flavor, aroma, and stimulating attributes of coffee cup quality as well as dietary benefits.

\section{Introduction}

Coffee is one of the most popular and widely consumed beverages all over the world. Its consumption is progressively increasing due to its distinct taste, aroma, and stimulating property [1]. Commercially, two coffee species, Coffea arabica (Arabica coffee) and Coffea canephora (Robusta coffee), are known [2]. Arabica coffee is characterized by having distinct flavor (tastes), aroma profiles, and stimulating effects, making the product attractive in the international markets [3-5]. In Ethiopia, Arabica coffee is grown in all parts of the country, i.e., at lowland, midland, and highland and, thus, expected to exhibit varying characteristics in terms of their physical and chemical compositions $[3,6]$.

The flavor, aroma profiles, and stimulating effects of coffee beans are greatly affected by the biochemical compositions of coffee beans, which are in turn influenced by geographical locations, climatic conditions, agricultural practices, genotypes, and the processing method used $[1,5,7,8]$. For instance, it was reported that Arabica coffee which is grown at high altitudes has better quality than lowland coffee. At high altitudes, coffee plants take longer time to mature, fully develop, and produce beans having good flavor and aroma $[4,5,7]$. Beside altitudes, the biochemical compositions of coffee beans are strongly influenced by environmental factors including the amount and distribution of rainfall and the physical and chemical characteristics of the soils $[1,9]$.

Coffee beans contain numerous biochemical components that markedly influence the quality of coffee cups. Some of these biochemicals are caffeine, trigonelline, chlorogenic acids, CGAs (3-caffeoylquinic acid, 3-CQA; 4caffeoylquinic acid, 4-CQA; and 5-CQA), sucrose, and lipids 
$[8,10,11]$. Each biochemical provides specific and/or additional contributions to the coffee quality attributes, bioactivities, and dietary source $[1,8,12]$. For instance, caffeine, which is an alkaloid and a nitrogenous secondary metabolite, provides bitterness attributes to the coffee quality. When consumed at moderate levels, it enhances alertness and concentration, energy availability, and physical performance as well as decreasing fatigue $[1,8]$. Nonetheless, too much consumption of caffeine may have negative outcomes such as addiction, depression, cardiovascular disease (cardiac arrhythmias and hypertensive palpitations), gastrointestinal disturbances, effects on bone status and calcium balance, bronchodilation, male fertility problems, and fetus-related issues during pregnancy $[8,12]$.

Similarly, CGAs such as 3-CQA, 4-CQA, and 5-CQA are other important phenolic components of coffee beans. They contribute flavors such as astringency, acidity, and bitterness in coffee beverage quality determination $[1,8]$. They are also used to protect the plant from abiotic stresses such as changes in temperature, water content, exposure to UV light levels, and deficiency of mineral nutrients [8]. Besides, they play great roles against attacks by pathogens, plant competition, and attraction of beneficial organisms such as pollinators, seed dispersers, and symbiotic microorganisms [13]. CGAs have antioxidative and radical scavenging properties as well as various biological effects such as protection from immunoprotective, cardiovascular, antihypertensive, anti-inflammatory, antiviral, antifungal, anticancer, antiaging, and neuroprotective actions [12].

Nicotinic acid (niacin or vitamin $B_{3}$ ) is another bioactive component of coffee beans. Its concentration in raw (green) coffee beans is relatively very small, but it increases after roasting process due to its formation from thermal decomposition of trigonelline $[1,2,8]$. Thus, coffee can be used as a significant dietary source of this vitamin. Daily consumption of coffee can supplement about one-third of the minimum dietary of adults' requirement of nicotinic acid $[8,14]$. Nicotinic acid plays significant roles in several oxidation and reduction reactions metabolic pathways and in lowering cholesterol. It has an ability to raise the concentration of high-density lipoprotein (HDL) in the plasma and thus can be used in hyperlipidemia, resulting in the reduction of the progression of atherosclerosis and risk of cardiovascular events. The deficiency of nicoticic acid results in pellagra, a syndrome characterized by dermatitis, diarrhea, dementia, and high mortality $[15,16]$.

Reported literature indicated that quality and biochemical compositions of coffee beans are highly influenced by environment, coffee accessions/varieties, growing altitudes, shade, harvesting periods, and so on [3-5]. However, in Ethiopia, the motherland and center of genetic diversity of Arabica coffee, much attention has not yet been given to how biochemical components of coffee beans such as caffeine, 5-CQA, and nicotinic acids vary among coffee varieties grown at different altitudes in different parts of the country. On the other hand, in the country, the demand for cultivation of better quality coffee is increasing from time to time. To satisfy the ever increasing demand of coffee cultivars, several improved Arabica coffee varieties that are characterized by better cup quality, yield, and tolerance to coffee diseases than the traditional ones have been released for cultivars since the early 1970s [17-19]. To complement the existing study, in this work, the influence of growing altitudes on concentrations of caffeine, 5-CQA, and nicotinic acid of four Coffee arabica le varieties grown in southwest of Ethiopia has been studied. Separation and quantitative determination of the three analytes were performed by HPLC-DAD.

\section{Materials and Methods}

2.1. Sample Collection and Pretreatment. Coffee bean samples were collected from three different farmlands owned by the Ethiopian Institute of Agricultural Research (EIAR) in Southwest of Ethiopia. These were from Gera Agricultural Research Subcenter and Jimma Agricultural Research Center which are located in Jimma Zone, Oromia Regional State, as well as from Teppi National Spies Research Center (TNSRC) which is located in Southern Nations, Nationalities, and Peoples' Region State (SNNPRS).

Four coffee varieties, namely, 74110, 7454, 7440, and 74112 , were purposively collected from the three farmlands in November and December 2017. The varieties identification code begin with " 74 " to indicate their cataloging and selection in 1974, while the last two or three numbers indicate their accession numbers [19]. From each farmland, $6 \mathrm{~kg}$ of red ripe coffee cherries (beans) was handpicked from 30 randomly selected coffee trees. Before pulping, immature cherries and other undesirable materials were removed. Samples were collected from Gera, Jimma, and Teppi research centers representing highland, midland, and lowland coffees, respectively, and details of the geographic locations and biophysical data of the study areas are presented in Table 1.

Wet processing procedure that involves pulping, fermentation, and drying of the collected samples was done at the sampling sites. Red ripened coffee cherries were manually pulped to separate the beans from the skin and pulp. The wet coffee parchment was kept for $40 \mathrm{~h}$ before the first washing was done. Then, the samples were soaked in water for $24 \mathrm{~h}$ before the final washing, to improve the quality and appearance of coffees by removing the brownish color originating from degraded sugars and polyphenols [20]. The washed samples were then spread thinly on raised metal wire bed and dried by sun, until the moisture contents of the beans were approximately $10.0-12.5 \%$ [5], which were within the safe moisture contents range (i.e., $8.0-12.5 \%$ ) for dried green coffee beans [21]. Finally, the dried coffee samples were placed in polyethylene bags and then transported to Jimma University Analytical Chemistry Research Laboratory. In the laboratory, all the samples were kept in a refrigerator below $4^{\circ} \mathrm{C}$ until analysis.

2.2. Roasting of Coffee Samples. The procedure reported by Barbosa and coworkers [11] was used for roasting coffee samples. Accordingly, after heating the roasting machine at 
TABle 1: Geographic locations and biophysical data of the study area.

\begin{tabular}{lccc}
\hline $\begin{array}{l}\text { Biophysical } \\
\text { data }\end{array}$ & $\begin{array}{c}\text { Highland } \\
\text { (Gera) }\end{array}$ & $\begin{array}{c}\text { Midland } \\
\text { (Jimma) }\end{array}$ & $\begin{array}{c}\text { Lowland } \\
\text { (Teppi) }\end{array}$ \\
\hline $\begin{array}{l}\text { Altitude (asl) } \\
\text { Mean }\end{array}$ & $1940-1960 \mathrm{~m}$ & $1750-1775 \mathrm{~m}$ & $1100-1200 \mathrm{~m}$ \\
temperature & $19^{\circ} \mathrm{C}-25^{\circ} \mathrm{C}$ & $20.5^{\circ} \mathrm{C}-27^{\circ} \mathrm{C}$ & $25^{\circ} \mathrm{C}-30^{\circ} \mathrm{C}$ \\
Rainfall & $1880-2080 \mathrm{~mm}$ & $1525-2000 \mathrm{~mm}$ & $800-2000 \mathrm{~mm}$ \\
Latitude & $6^{\circ} 30^{\prime} \mathrm{N}$ & $7^{\circ} 40^{\prime} \mathrm{N}$ & $9^{\circ} 08^{\prime} \mathrm{N}$ \\
Longitude & $32^{\circ} 15^{\prime} \mathrm{E}$ & $36^{\circ} 50^{\prime} \mathrm{E}$ & $37^{\circ} 13^{\prime} \mathrm{E}$ \\
\hline
\end{tabular}

asl: above sea level.

about $200^{\circ} \mathrm{C}, 100 \mathrm{~g}$ of dried coffee beans was added and roasted for about $8 \mathrm{~min}$ on average. The roasted sample was transferred to a tray for cooling. Subsequently, after removing the loose silver skins by blowing, the roasted sample was ground using coffee grinding machine (ADE Germany GmbH \& Co. KG, Hamburg, Germany).

2.3. Chemicals and Reagents. Chemicals and reagents used were of analytical grade, and solvents were of HPLC grade. Analytical grade standards of 5-CQA (99.9\%), caffeine (99.9\%), and nicotinic acid (99.9\%) were obtained from Sigma Aldrich (St. Louis, MO, USA). HPLC grades of acetonitrile and glacial acetic acid were obtained from Carlo Erba Reagents S.A.S. (Mumbai, India). Ultrapure water was used throughout the work.

Individual stock solution containing $1000 \mathrm{mg} / \mathrm{L}$ of caffeine, 5-CQA, and nicotinic acid was prepared by dissolving in acetonitrile. Intermediate solution containing $100 \mathrm{mg} / \mathrm{L}$ of each standard was prepared in the same solvent. Working solutions were then prepared by diluting the intermediate solution with ultrapure water. For construction of external calibration curves, series of solutions of each analyte were prepared by serial dilution. Stock and intermediate standard solutions were kept in the refrigerator below $4^{\circ} \mathrm{C}$ when not used.

2.4. Instrumentation and Apparatus. Chromatographic separation was performed using an Agilent HPLC 1260 Infinity system (Agilent Technologies, Germany) equipped with a quaternary pump system (maximum flow rates, $10 \mathrm{~mL} / \mathrm{min}$ ), online degasser, autosampler, column thermostat, and diode array detector (DAD). Agilent ChemStation B.04.02 SP2 was used for data acquisition and processing. Spherisorb ODS-1 column $(150 \mathrm{~mm} \times 4.6 \mathrm{~mm}$; $5 \mu \mathrm{m})$ was used for chromatographic separation of the analytes. Coffee grinding machine was used for grinding coffee samples.

2.5. Chromatographic Conditions. Chromatographic conditions reported by Dias and Benassi [22] were adopted for separation of the analytes. Accordingly, separation was performed using binary solvents containing acetic acid: $\mathrm{H}_{2} \mathrm{O}$ $(5: 95, \mathrm{v} / \mathrm{v})$ (as solvent A) and acetonitrile (as solvent B). Isocratic elution with solvent $A$ : solvent $B$ ratio of $95: 5, \mathrm{v} / \mathrm{v}$; column temperature, $30{ }^{\circ} \mathrm{C}$; elution flow rate, $1.0 \mathrm{~mL} / \mathrm{min}$; and injection volume, $20 \mu \mathrm{L}$ were used throughout the analysis. The target analytes were analyzed at various monitoring wavelengths: $272 \mathrm{~nm}, 320 \mathrm{~nm}$, and $260 \mathrm{~nm}$ for caffeine, 5-CQA, and nicotinic acid, respectively. The calibration curves were constructed as peak area versus the concentration of the analytes. Then, the levels of caffeine, chlorogenic acid, and nicotinic acid in coffee samples were calculated using the regression equation of the curves.

2.6. Sample Preparation. Prior to their chromatographic separation and quantitative determination, the target analytes were extracted from coffee samples using the method reported by Dias and Benassi [22]. Accordingly, $0.5 \mathrm{~g}$ roasted or raw ground coffee sample was dissolved in $30 \mathrm{~mL}$ solvent containing water and acetonitrile (ratio: 95:5, v/v). The content was placed in water bath at $80^{\circ} \mathrm{C}$ and boiled for 10 min. After filtration with Whatman No. 1 filter paper, $5 \mathrm{~mL}$ of the filtrate was taken into $25 \mathrm{~mL}$ volumetric flask filled to the mark with the water: acetonitrile $(95: 5 \mathrm{v} / \mathrm{v})$ solvent. Finally, about $2 \mathrm{~mL}$ of the resulting solution was filtered with $0.22 \mu \mathrm{m}$ nylon syringe filter into $2 \mathrm{~mL}$ autosampler vial to inject $20 \mu \mathrm{L}$ of the extract into the HPLCDAD system.

2.7. Evaluation of Method Performance. Performance characteristics of the utilized method were validated in terms of linear dynamic range (LDR), accuracy, precision, limits of detection (LOD), and quantification (LOQ) [7]. The LDR of each analyte was validated by constructing calibration curves using series of solutions that produce coefficient of determination $\left(r^{2}\right)$ of 0.99 or better. LOD and LOQ were determined as the lowest concentrations yielding 3 and 10 times signal-to-noise ratio $(\mathrm{S} / \mathrm{N})$ of the chromatogram, respectively [23]. Precision was evaluated by replicate analyses, i.e., by preparing duplicate samples and injecting each sample in duplicate to have the total of four replicate measurements $(n=4)$. Recovery study $(\% \mathrm{R})$ was performed by spiking known concentrations of the target analytes, i.e., $5 \mathrm{mg} / \mathrm{L}$ of each analyte, onto the coffee sample. Table 2 shows analytical performance of the employed method.

2.8. Statistical Analysis. Descriptive statistics were used for reporting and comparing the obtained findings: mean and $S D$, Student's $t$-test, and Person correlation coefficient as well as two-way ANOVA. Statistical analyses were performed by SAS 9.0 software. All results were reported as the mean \pm SD of a replicate of measurements $(n=4)$.

\section{Results and Discussion}

Separation and determination of nicotinic acid, caffeine, and 5-CQA were performed using HPLC-DAD at monitoring wavelengths of $260 \mathrm{~nm}, 272 \mathrm{~nm}$, and $320 \mathrm{~nm}$, respectively. Figure 1 shows their representative chromatograms obtained from analysis of a raw coffee sample. 
TABLE 2: Analytical performance characteristics of the adopted method.

\begin{tabular}{lcccccc}
\hline Analyte & $\begin{array}{c}\mathrm{LDR} \\
(\mathrm{mg} / \mathrm{L})\end{array}$ & $r^{2}$ & $\begin{array}{c}\text { LOD } \\
(\mathrm{mg} / \mathrm{L})\end{array}$ & $\begin{array}{c}\text { LOQ } \\
(\mathrm{mg} / \mathrm{L})\end{array}$ & Raw & Roasted \\
\hline Caffeine & $0.20-10.00$ & 0.998 & 0.07 & 0.20 & $95 \pm 0.80$ & $88 \pm 2.10$ \\
5-CQA & $0.26-10.00$ & 0.997 & 0.09 & 0.26 & $101 \pm 0.30$ & $95 \pm 0.60$ \\
Nicotinic acid & $0.23-10.00$ & 0.996 & 0.08 & 0.23 & $100 \pm 3.40$ & $99 \pm 0.12$ \\
\hline
\end{tabular}

The obtained concentrations of the target analytes in raw (green) and roasted coffee varieties collected from different altitudes are displayed in Table 3.

As can be seen from Table 3, the caffeine contents of green coffee varieties varied within the following ranges: $15.14-19.89 \mathrm{mg} / \mathrm{g}$ (lowland), $13.83-16.07 \mathrm{mg} / \mathrm{g}$ (midland), and $12.34-13.58 \mathrm{mg} / \mathrm{g}$ (highland). Similarly, in roasted coffee beans they varied within the following ranges: $19.21-20.88 \mathrm{mg} / \mathrm{g}$ (lowland), $14.21-18.62 \mathrm{mg} / \mathrm{g}$ (midland), and $13.93-17.22 \mathrm{mg} / \mathrm{g}$ (highland) coffee varieties. The lowest caffeine contents were observed in highland coffee varieties $74110(12.34 \mathrm{mg} / \mathrm{g})$ and $74112(13.93 \mathrm{mg} / \mathrm{g})$ for green and roasted coffee beans, respectively, whereas for both green and roasted beans the highest concentrations were observed in the lowland coffee variety 7454 . Regardless of the studied coffee varieties, caffeine contents of green and roasted beans decrease as their growing altitude increases. Correlation study between caffeine content and altitude also showed that they have strong negative correlation, i.e., $r$ values -0.981 and -0.990 for green and roasted coffee beans, respectively. Moreover, it has been observed that the impacts of growing altitudes on the concentrations of caffeine among coffee varieties are different. Two-way ANOVA at $p<0.5$ revealed that caffeine contents of green and roasted coffee varieties are significantly affected by coffee varieties and growing altitude.

Previously reported studies also showed that the caffeine content of coffee varies with coffee varieties and growing altitudes [3-5, 24]. A study conducted on 99 Coffee arabica progenies, which are grown in different geographical locations of Ethiopia, showed that their caffeine contents varied from 4.6 to $28.00 \mathrm{mg} / \mathrm{g}$ [20]. Tolessa et al. [4] and Worku et al. [5] also reported similar findings that the caffeine contents of coffee are highly influenced by altitude.

It was also observed that, for each coffee variety studied, roasted coffee beans have higher caffeine content than the corresponding green coffee beans, as has been also reported by Sunarharum et al. [1] and Tolassa et al. [3]. During roasting processes, different chemicals such as water, carbon dioxide, and volatile compounds are lost from coffee beans, resulting in enrichment of thermally stable substances such as caffeine in the roasted beans. Except for coffee variety 74112 at mid- and highlands, a statistical $t$-test at $p<0.05$ also demonstrated the presence of significant differences between the caffeine contents of roasted and native green coffee beans. However, in general, the caffeine contents of the studied samples, both in green and roasted coffee samples, are within the caffeine content range of Coffee arabica reported in the literature $[8,20]$.
5-CQA is another important biochemical used for verification of coffee varieties. In the current study, its concentrations in green coffee were in the following ranges: $27.17-34.56 \mathrm{mg} / \mathrm{g}$ (in highland), $30.72-34.62 \mathrm{mg} / \mathrm{g}$ (in midland); and $32.06-39.18 \mathrm{mg} / \mathrm{g}$ (in lowland) coffee varieties. Likewise, in roasted coffee beans, its concentrations were in the following ranges: $8.56-12.59 \mathrm{mg} / \mathrm{g}$, $12.51-15.25 \mathrm{mg} / \mathrm{g}$, and $14.19-16.21 \mathrm{mg} / \mathrm{g}$ for coffee varieties collected from highland, midland, and lowland, respectively. In both green and roasted coffee beans, its lowest concentrations were recorded in the same coffee variety 7454, which was collected from highland farmland. However, the highest concentration was detected in coffee varieties collected from lowland, i.e., in coffee varieties 7454 for green and 7440 for roasted coffee samples. For each coffee variety, the lowest concentrations of 5-CQA were observed in highland coffees and the highest were determined in lowland coffee beans. Correlation studies also demonstrated that there are strong negative relationship, i.e., $r$ values -0.917 and -0.727 for green and roasted coffee beans, respectively, between coffee variety and altitude. Statistical analysis by two-way ANOVA at $p<0.05$ also indicated that the concentrations of 5-CQA are significantly varied with coffee varieties and altitudes. The variation of concentrations of 5-CQA with altitudes as well as coffee varieties were also reported in the literature $[3,21,24]$.

Besides, for all studied coffee varieties, the levels of 5CQA decrease as the growing altitude rises. Roasted beans contain lower contents of 5-CQA than the native green coffee beans. During roasting, $49.43-71.15 \%$ of the compound was lost, indicating its thermal degradation in the process. It has also reported that CGAs are thermally instable and, thus, $60.90 \%-96.50 \%$ of the compounds can be lost during roasting of Arabica coffee [1, 8, 21]. Statistical $t$ test at $p<0.05$ has also demonstrated that there are significance differences between the contents of 5-CQA in green coffee and its corresponding roasted samples. Although, the concentrations of CGAs decrease upon roasting, a cup $(200 \mathrm{~mL})$ of Arabica coffee brew contains 70-200 mg of CGAs, indicating that coffee beverage is one of the main sources of CGAs compounds in human diet. CGAs, like 5CQA, provide acidity, astringency, and bitterness attributes to coffee cups and are used as antioxidants for human health $[1,13,14]$.

The nicotinic acid contents of green coffee varieties varied within the ranges $7.13-8.25 \mathrm{mg} / \mathrm{g}, 7.38-8.34 \mathrm{mg} / \mathrm{g}$, and $8.08-10.16 \mathrm{mg} / \mathrm{g}$, and in roasted coffee they were in the ranges $6.55-7.55 \mathrm{mg} / \mathrm{g}, 6.66-7.98 \mathrm{mg} / \mathrm{g}$, and $7.92-8.76 \mathrm{mg} / \mathrm{g}$ in lowland, midland, and highland, respectively. In green coffee samples, the smallest and highest concentrations of the compound were observed in coffee varieties 7440 (lowland) and 7454 (highland), whereas in roasted samples the lowest and highest concentration of nicotinic acid were found in the same coffee variety, 7454, collected from lowland and highland farmlands, respectively. In contrast to caffeine and 5-CQA, the contents of nicotinic acid in both green and roasted coffee beans are relatively increased as altitude increases. The correlation study between coffee 


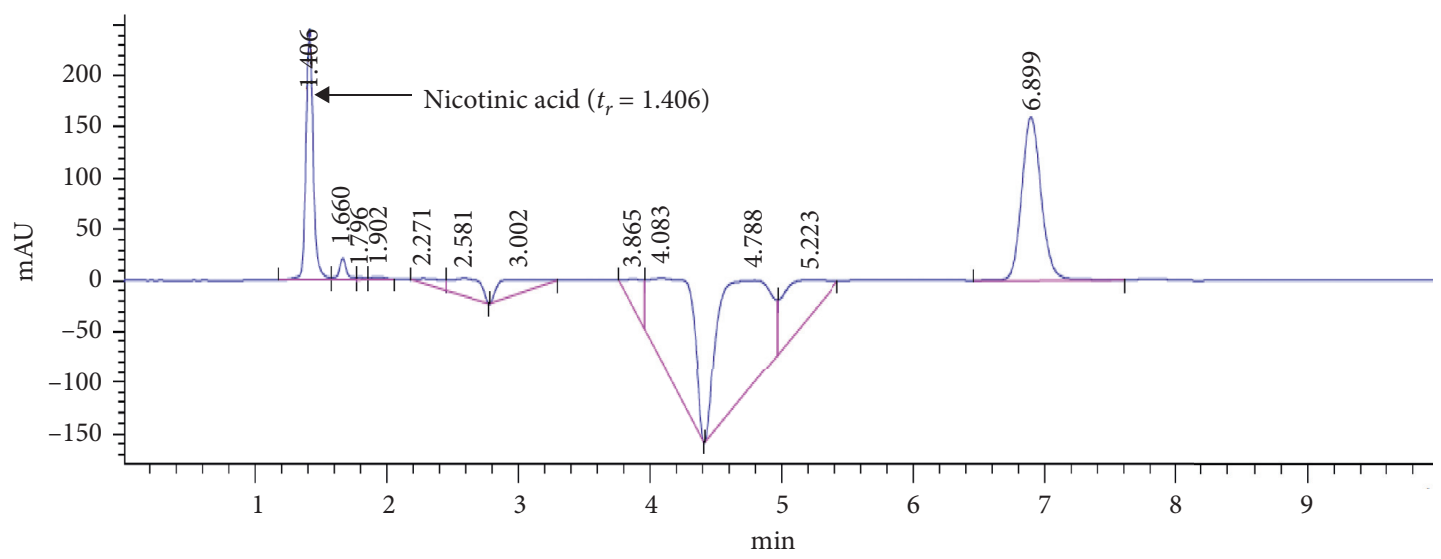

(a)

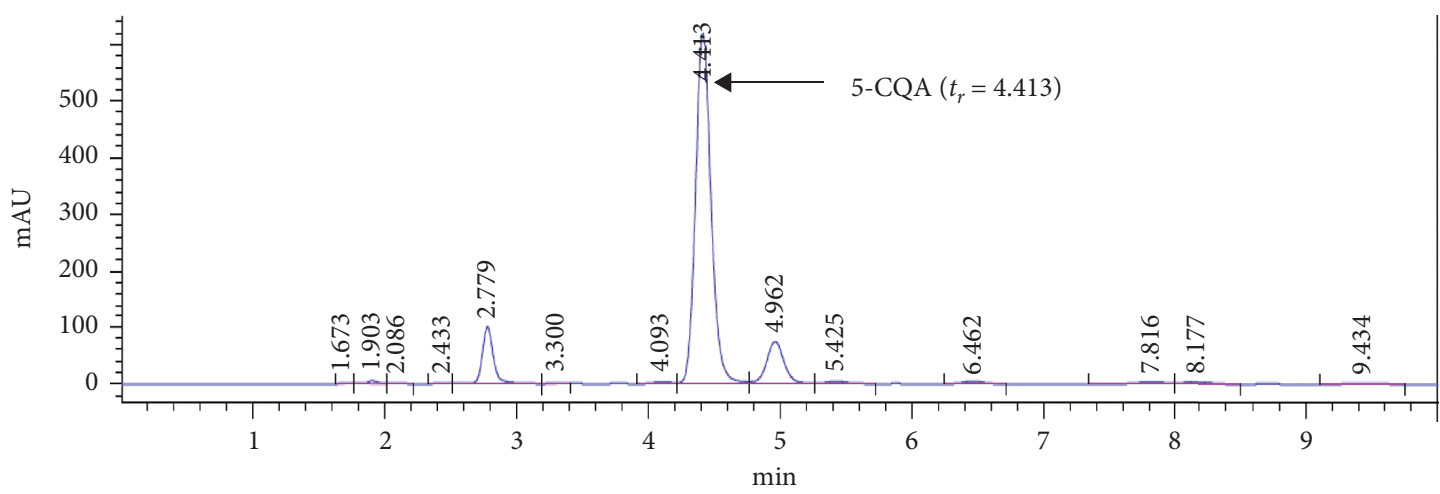

(b)

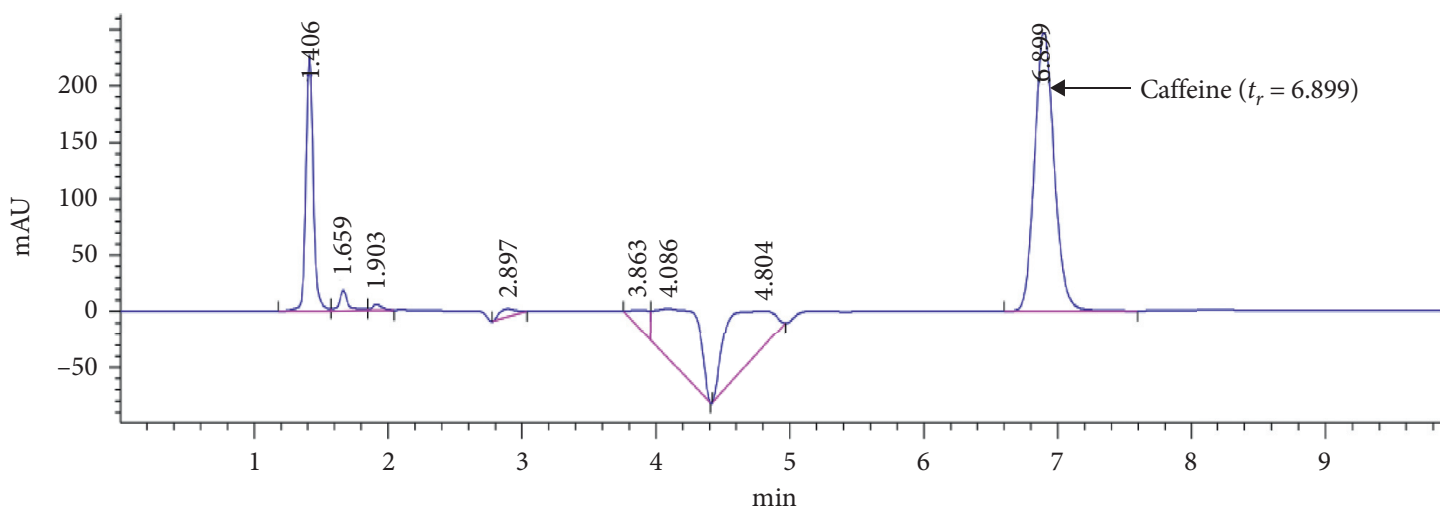

(c)

FIGURE 1: Representative chromatograms of nicotinic acid, caffeine, and chlorogenic acid obtained from green coffee variety 74110.

varieties and altitudes also indicated that they have strong positive correlation: 0.845 and 0.997 for green and roasted coffees, respectively. Statistical evaluation using two-way ANOVA $(p<0.05)$ also revealed the presence of significant differences in nicotinic acid contents of both green and roasted coffee varieties with growing altitudes.

Irrespective of their varieties and growing altitudes, roasted coffee beans showed relatively lower concentrations of nicotinic acids than the corresponding green coffee beans. The obtained results agreed with some reported literature findings $[7,25]$. However, several studies indicated that roasted coffee beans contain higher concentrations of nicotinic acids than green coffee beans, due to their formation from the decomposition of trigonelline during roasting processes [7, 8, 14]. The concentrations determined in the present study were found to be higher than the values reported in the literature $[2,9,12,14]$. These variations might be exhibited due to the difference of coffee origin and/or species as well as the degree of roasting, which greatly influences the level of nicotinic acids in the coffee $[7,8]$. On the other hand, the presence of higher concentrations of nicotinic acid could be beneficial to contribute the nicotinic acid (vitamin $B_{3}$ ) requirement in diet. 
TABLE 3: Concentrations in mg/g of caffeine, 5-CQA, and nicotinic acid in green and roasted coffee varieties grown at different altitudes of Southwest Ethiopia (mean $\pm \mathrm{SD}, n=4$ ).

\begin{tabular}{|c|c|c|c|c|c|c|c|}
\hline \multirow{2}{*}{ Altitude, asl } & \multirow{2}{*}{ Coffee variety } & \multicolumn{2}{|c|}{ Caffeine } & \multicolumn{2}{|c|}{ 5-CQA } & \multicolumn{2}{|c|}{ Nicotinic acid } \\
\hline & & Green & Roasted & Green & Roasted & Green & Roasted \\
\hline \multirow{5}{*}{$\begin{array}{l}\text { Gera } \\
1940-1960 \mathrm{~m}\end{array}$} & 74110 & $12.34 \pm 0.08$ & $17.22 \pm 0.76$ & $29.72 \pm 0.71$ & $11.48 \pm 0.46$ & $8.16 \pm 0.04$ & $7.92 \pm 0.18$ \\
\hline & 7454 & $13.58 \pm 0.15$ & $16.92 \pm 1.37$ & $27.17 \pm 0.38$ & $8.56 \pm 0.02$ & $10.16 \pm 0.75$ & $8.76 \pm 0.11$ \\
\hline & 7440 & $13.10 \pm 0.45$ & $14.49 \pm 1.69$ & $30.40 \pm 1.44$ & $8.77 \pm 0.38$ & $8.53 \pm 0.45$ & $8.44 \pm 0.08$ \\
\hline & 74112 & $13.30 \pm 0.77$ & $13.93 \pm 0.29$ & $34.56 \pm 2.09$ & $12.59 \pm 0.21$ & $8.08 \pm 0.16$ & $7.98 \pm 0.15$ \\
\hline & Mean & $13.08 \pm 0.63$ & $15.99 \pm 2.19$ & $31.50 \pm 3.05$ & $13.03 \pm 3.36$ & $8.73 \pm 1.12$ & $7.84 \pm 0.69$ \\
\hline \multirow{5}{*}{$\begin{array}{l}\text { Jimma } \\
\text { 1750-1775 m }\end{array}$} & 74110 & $14.51 \pm 0.08$ & $18.62 \pm 0.47$ & $31.34 \pm 1.67$ & $13.16 \pm 0.43$ & $8.15 \pm 0.19$ & $7.74 \pm 0.45$ \\
\hline & 7454 & $16.07 \pm 0.58$ & $17.72 \pm 1.46$ & $33.85 \pm 2.26$ & $15.25 \pm 1.19$ & $8.34 \pm 1.57$ & $7.98 \pm 0.69$ \\
\hline & 7440 & $13.83 \pm 0.54$ & $16.55 \pm 0.39$ & $30.72 \pm 0.64$ & $12.51 \pm 0.21$ & $7.38 \pm 0.96$ & $7.23 \pm 0.33$ \\
\hline & 74112 & $13.87 \pm 0.53$ & $14.21 \pm 0.71$ & $34.62 \pm 0.07$ & $13.61 \pm 0.36$ & $7.87 \pm 0.49$ & $6.66 \pm 0.03$ \\
\hline & Mean & $14.48 \pm 1.34$ & $16.43 \pm 1.58$ & $33.15 \pm 2.18$ & $12.02 \pm 2.49$ & $7.91 \pm 0.46$ & $7.33 \pm 0.67$ \\
\hline \multirow{5}{*}{$\begin{array}{l}\text { Teppi } \\
1100-1200 \mathrm{~m}\end{array}$} & 74110 & $15.14 \pm 0.39$ & $20.28 \pm 0.63$ & $32.16 \pm 2.11$ & $14.82 \pm 1.50$ & $7.59 \pm 0.68$ & $7.55 \pm 0.34$ \\
\hline & 7454 & $19.89 \pm 0.29$ & $20.88 \pm 0.14$ & $39.18 \pm 0.24$ & $15.27 \pm 0.01$ & $8.25 \pm 0.27$ & $6.34 \pm 0.03$ \\
\hline & 7440 & $15.60 \pm 0.96$ & $20.54 \pm 0.09$ & $32.06 \pm 1.76$ & $16.21 \pm 0.10$ & $7.13 \pm 0.53$ & $7.36 \pm 0.88$ \\
\hline & 74112 & $15.67 \pm 0.33$ & $19.21 \pm 0.66$ & $33.62 \pm 0.88$ & $14.19 \pm 0.32$ & $7.54 \pm 0.71$ & $6.55 \pm 0.33$ \\
\hline & Mean & $16.42 \pm 2.19$ & $20.23 \pm 0.04$ & $33.23 \pm 3.95$ & $14.05 \pm 1.31$ & $7.63 \pm 0.05$ & $6.23 \pm 0.83$ \\
\hline \multicolumn{2}{|c|}{ Correlation coefficient $(r)$} & -0.981 & -0.990 & -0.917 & -0.727 & 0.845 & 0.997 \\
\hline
\end{tabular}

\section{Conclusions}

In the present study, the contents of caffeine, 5-CQA, and nicotinic acid in four Arabica coffee varieties, namely, $74110,7454,7440$, and 74112, which are grown at different altitudes in southwest Ethiopia, were investigated. Red ripened coffee cherry samples were purposively collected from Gera, Jimma, and Teppi EIAR owned farmlands, which are located in high, mid, and low altitudes, respectively, and then wet coffee processing method was employed for pretreatment of the samples. Before performing separation and quantitative determination by HPLC-DAD, the target analytes were extracted by $\mathrm{H}_{2} \mathrm{O}$ : acetonitrile $(95: 5, \mathrm{v} / \mathrm{v})$ solvent from both green and roasted coffee bean samples. The findings demonstrated that concentrations of both caffeine and 5-CQA decrease against the growing altitudes, whereas the concentrations of nicotinic acid increase as altitudes rise up, in both green and roasted coffee beans. Nevertheless, roasted coffee beans contain higher concentrations of caffeine than green beans. However, the levels of 5-CQA were significantly lower in the roasted coffee than those in the native green beans. It was also observed that the studied coffee varieties have significantly high nicotinic acid contents, indicating their importance as potential source in diet. Generally, the study results indicated that the contents of caffeine, 5-CQA, and nicotinic acid of coffee beans vary with coffee varieties and their growing altitudes, indicating that the inherent nature of coffee beans and their growing altitudes are crucial parameters that affect the biochemical compositions of the products.

\section{Data Availability}

The compiled excel data used to support the findings of this study are available from the corresponding author upon request.

\section{Conflicts of Interest}

All the authors declare that they have no conflicts of interest regarding the publication of this paper.

\section{Acknowledgments}

This work was funded by Jimma Agricultural Research Center, Jimma, Ethiopia. The authors appreciate Jimma University Laboratory of Drug Quality (JuLaDQ), Department of Pharmacy, for providing laboratory space and facilities.

\section{References}

[1] W. B. Sunarharum, D. J. Williams, and H. E. Smyth, "Complexity of coffee flavor: a compositional and sensory perspective," Food Research International, vol. 62, pp. 315325, 2014.

[2] S. Casal, M. B. Oliveira, and M. A. Ferreira, "Development of an HPLC/diode-array detector method for simultaneous determination of trigonelline, nicotinic acid, and caffeine in coffee," Journal of Liquid Chromatography and Related Technologies, vol. 21, no. 20, pp. 3187-3195, 1998.

[3] K. Tolessa, J. D'heer, L. Duchateau, and P. Boeckx, "Influence of growing altitude, shade and harvest period on quality and biochemical composition of Ethiopian specialty coffee," Journal of the Science of Food and Agriculture, vol. 97, no. 9, pp. 2849-2857, 2017.

[4] M. Hagos, M. Redi-Abshiro, D. S. Chandravanshi, E. Ele, A. M. Mohammed, and H. Mamo, "Correlation between caffeine contents of green coffee beans and altitudes of the coffee plants grown in southwest Ethiopia," Bulletin of the Chemical Society of Ethiopia, vol. 31, no. 1, pp. 13-25, 2018.

[5] M. Worku, B. de Meulenaer, L. Duchateau, and P. Boeckx, "Effect of altitude on biochemical composition and quality of green Arabica coffee beans can be affected by shade and postharvest processing method," Food Research International, vol. 105, pp. 278-285, 2018. 
[6] A. Mintesnot and N. Dechassa, "Effect of altitude, shade, and processing methods on the quality and biochemical composition of green coffee beans in Ethiopia," East African Journal of Sciences, vol. 12, no. 2, pp. 87-100, 2018.

[7] G. Caprioli, M. Cortese, F. Maggi et al., "Quantification of caffeine, trigonelline and nicotinic acid in espresso coffee: the influence of espresso machines and coffee cultivars," International Journal of Food Science and Nutrition, vol. 65, no. 4, pp. 465-469, 2014.

[8] B. Cheng, A. Furtado, H. E. Smyth, and R. J. Henry, "Influence of genotype and environment on coffee quality," Trends in Food Science \& Technology, vol. 57, pp. 20-30, 2016.

[9] C. S. G. Kitzberger, M. B. D. S. Scholz, and M. D. T. Benassi, "Bioactive compounds content in roasted coffee from traditional and modern Coffea Arabica cultivars grown under the same edapho-climatic conditions," Food Research International, vol. 61, pp. 61-66, 2014.

[10] G. Navarra, M. Moschetti, V. Guarrasi, M. Mangione, V. Militello, and M. Leonel, "Simultaneous determination of caffeine and chlorogenic acids in green coffee by UV/vis spectroscopy," Journal of Chemistry, vol. 1017, Article ID 6435086, 2017.

[11] M. D. S. G. Barbosa, M. B. D. S. Scholz, C. S. G. Kitzberger, and M. D. T. Benassi, "Correlation between the composition of green Arabica coffee beans and the sensory quality of coffee brews," Food Chemistry, vol. 292, pp. 275-280, 2019.

[12] C. Ciaramelli, A. Palmioli, and C. Airoldi, "Coffee variety, origin and extraction procedure: implications for coffee beneficial effects on human health," Food Chemistry, vol. 278, pp. 47-55, 2019.

[13] M. C. Monteiro and A. Farah, "Chlorogenic acids in Brazilian Coffea arabica cultivars from various consecutive crops," Food Chemistry, vol. 134, no. 1, pp. 611-614, 2012.

[14] D. Perrone, C. M. Donangelo, and A. Farah, "Fast simultaneous analysis of caffeine, trigonelline, nicotinic acid and sucrose in coffee by liquid chromatography-mass spectrometry," Food Chemistry, vol. 110, no. 4, pp. 1030-1035, 2008.

[15] K. Kraemer, R. D. Semba, M. Eggersdorfer, and D. A. Schaumberg, "Introduction: the diverse and essential biological functions of vitamins," Annals of Nutrition and Metabolism, vol. 61, no. 3, pp. 185-191, 2012.

[16] J. G. Dórea and T. H. M. da Costa, "Is coffee a functional food?" British Journal of Nutrition, vol. 93, no. 6, pp. 773-782, 2005.

[17] T. Kufa, A. Ayano, A. Yilma, T. Kumela, and W. Tefera, "The contribution of coffee research for coffee seed development in Ethiopia," E3 Journal of Agricultural Research and Development, vol. 1, no. 1, 2011.

[18] S. Diro, B. Erko, and K. Fikirie, "Production and adoption constraints of improved coffee varieties in Jimma zone, Southwest Ethiopia," Journal of Scientific Agriculture, vol. 3, pp. 33-40, 2019.

[19] D. Teferi, "Achievements and prospects of coffee research in Ethiopia: a review," International Journal of Research Studies in Agricultural Sciences, vol. 5, no. 11, pp. 41-51, 2019.

[20] M. B. Silvarolla, P. Mazzafera, and M. M. A. D. Lima, "Caffeine content of Ethiopian Coffea arabica beans," Genetics and Molecular Biology, vol. 23, no. 1, pp. 213-215, 2000.

[21] P. Wongsa, N. Khampa, S. Horadee, J. Chaiwarith, and N. Rattanapanone, "Quality and bioactive compounds of blends of Arabica and Robusta spray-dried coffee," Food Chemistry, vol. 283, pp. 579-587, 2019.

[22] R. Dias and M. Benassi, "Discrimination between arabica and robusta coffees using hydrosoluble compounds: is the efficiency of the parameters dependent on the roast degree?" Beverages, vol. 1, no. 3, pp. 127-139, 2015.

[23] N. C. Bicho, A. E. Leitão, J. C. Ramalho, N. B. de Alvarenga, and F. C. Lidon, "Identification of chemical clusters discriminators of arabica and robusta green coffee," International Journal of Food Properties, vol. 16, no. 4, pp. 895-904, 2013.

[24] C.-L. Ky, J. Louarn, S. Dussert, B. Guyot, S. Hamon, and M. Noirot, "Caffeine, trigonelline, chlorogenic acids and sucrose diversity in wild Coffea arabica L. and C. canephora P. accessions," Food Chemistry, vol. 75, no. 2, pp. 223-230, 2001.

[25] M. Minamisawa, S. Yoshida, and N. Takai, "Determination ofbiologically active substances in roasted coffee uing diodearray HPPLC system," Analytical Chemistry, vol. 20, pp. 325-328, 2014. 